\title{
Familienrechtliche Berechnungsprogramme
}

"Versorgungsausgleich" und „Unterbalt” des OSX Software GmbH

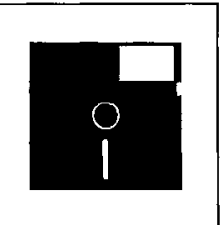

Wolfgang Michel

I. „Versorgungsausgleich”

Programmversionen 4.0, 4.1, beide 1. Halbjabr 1992

Nach der Beschreibung im Handbuch bietet das Programm eine Erfassung der Versorgungsanwartschaften der Berechtigten und Verpflichteten aus der gesetzlichen Rentenversicherung, der Beamtenversorgung, der $\mathrm{Zu}$ satzversorgung des öffentlichen Dienstes sowie der betrieblichen Altersversorgung. Soweit erforderlich, dynamisiert es die beiden letztgenannten Anwartschaften, wobei die dazu notwendigen $\mathrm{Da}$ ten und Werte ermittelt werden. Die Barwertfaktoren können entsprechend der Versorgungsart ausgewählt werden. Das Programm „vollzieht” den Versorgungsausgleich, ermittelt die Ausgleichspflichtigen sowie die Ausgleichswerte, überprüft die Höchstbetragsgrenze und ermittelt den Gegenstandswert. Beim Vollzug des Versorgungsausgleichs sind auch Optionen für einen "Ausgleich in sonstiger Weise" gemäß $\$ \$ 1$, III, 3 b I Nr. 1 und 2 VAHRG sowie „schuldrechdicher Versorgungsausgleich” gegeben.

\section{Dokumentation}

Das Handbuch hat eine ansprechende Aufmachung, einen gut lesbaren Druck und eine übersichtliche Gestaltung. Sein Inhalt ist auch für weniger computergeübte Juristen ohne Schwierigkeiten verständlich. Es verzichtet weitgehend auf EDV-Fachchinesisch. Die vorliegende Fassung war, im Gegensatz zum Programm, noch nicht dem ab dem 01.01.1992 in Kraft getretenen neuen Rentenversicherungsrecht und den damit verbundenen umfangreichen Änderungen des
Versorgungsausgleichsrechts angepaßt. So wird etwa noch die Markierung der „bagatellisierungsfähigen Beträge" angekündigt, während das Programm dem Wegfall der Bagatellklausel durch die Neuregelung selbstverständlich Rechnung trägt. Die neue Version des Programms eröffnet auch die Möglichkeit, den Versorgungsausgleich - Rentenversicherung und Beamtenversorgung - gemäß $\$ 2$ I Ziff. 1 a VAÜG für das Beitrittsgebiet zu berechnen, auch das steht natürlich noch nicht im Handbuch. $\mathrm{Als}$ gravierend kann die noch nicht erfolgte Aktualisierung jedoch kaum angesehen werden, denn die zur Anwendung des Programms notwendigen Informationen sind auch in der vorliegenden älteren Fassung enthalten. Im übrigen hat der Verlag der neuen Programmversion ein umfangreiches "readme” beigegeben, das auf alle diese Punkte sowie auf zusätzlich vorgenommene Bedienungsänderungen gut verständlich hinweist.

\section{Installation und Bedienung}

Installation und Bedienung des Programms bereiten, nicht zuletzt wegen der gut verständlichen Einweisung durch das Handbuch in Verbindung mit dem „readme”, keine beziehungsweise keine größeren Probleme. Bei einer Konstellation "stürzte das Programm (Version 4.1) allerdings ab". Wenn man nach durchgeführter Dynamisierung die Tafel „Dynamisierung der Anwartschaft aus der betrieblichen Altersversorgung" mit den angewendeten Rechengrößen und dem Ergebnis auf dem Bildschirm hat und dann $<$ F1> „Hilfe" bedient, bekommt man - meist - den Hinweis, daß für diese Ebene kein Hilfe-Text gefunden wurde mit dem Zusatz „Interne Hilfe - Nr. 9320"; bisweilen führt das aber auch dazu, daß nichts mehr geht. Das Bild begann sich manchmal sogar unter Pieptönen langsam Zeile um Zeile zu löschen. (Auf einen entsprechenden Hinweis des Rezensenten hat der Verlag diesen Fehler in der allerneusten Programmversion (4.1a) behoben; bei Betätigung der $<\mathrm{F} 1>$-Taste bekommt man jetzt den - zutreffenden - Hinweis, daß man mit der <Enter $>$-Taste oder der $<F 8>$-Taste zurück zur Erfassungsmaske gelange). Negativ erscheint auch die nichtkonsistente Belegung der Funktionstasten. Das Sichbewegen im Programm bedarf, auch nach den Verbesserungen in der Version 4.1 gegenüber der Version 4.0, immer noch einer gewissen Gewöhnung.

Nach Aufruf des Programms erscheint das aktuelle Datum als Berechnungsdatum, das durch Überschreiben geändert werden kann (Abb. 1, s. Folgeseite).

\section{Plausibilitätsprüfung und Programmfehler}

Das eingegebene Datum wird auf seine Plausibilität überprüft. Diese Überprüfung offenbart einige Besonderheiten. Bei Eingabe „Berechnungsdatum 01.03.99" erfuhr man bei der zunächst vorliegenden Version 4.0, daß dieses Datum außerhalb der Gültigkeit des Programms lieǵe und man 
sich an die OSX Software GmbH zwecks Erwerbs eines Updates des Programms zur Berechnung des „Unterhalts” wenden solle, $\mathrm{da}$ die vorliegende Version Tabellenwerte vom 01.01.1989 bis zum 30.06.1992 enthalte.

Dieser Fehler ist ab Version 4.1 insoweit behoben, als jetzt auf den Erwerb eines Updates des Programms „Versorgungsausgleich" hingewiesen wird.

Bei Eingabe des Berechnungsdatums 01.13.1991 zeigt der Bildschirm den Hinweis: „Bitte geben Sie ein Datum in gewohntem Format ein, z. B. 28.10.1980!". Ein Hinweis darauf, daß es sich nicht um die falsche Eingabe eines möglichen, sondern um die Eingabe eines unmöglichen $\mathrm{Da}$ tums handelt, erfolgt nicht. Auf jeden Fall wird man aber darauf hingewiesen, daß man etwas falsch gemacht hat.

Bei Angabe des Anfangs der Ehezeit etwa mit dem 02.02.1990 erscheint der rechtlich zutreffende Hinweis auf $\$ 1587$ Abs. 2 BGB, jedoch mit dem grammatikalisch verunglückten Zusatz: „Das Programm setzt Ihr eingegebenes Datum automatisch auf den 1. des jeweiligen Monats ge. setzt!". Gibt man das Ende der Ehezeit nicht mit dem Monatsende an, wird man hingegen nicht nur rechtlich zutreffend, sondern auch grammatikalisch richtig darauf hingewiesen, daß der gemäß $\ 1587$ Abs. 2 BGB maßgebliche Zeitpunkt vom Programm automatisch bestimmt werde.

Gibt man bei der Dynamisierung von Anwartschaften aus der betrieblichen Altersversorgung als Geburtsdatum des Anwartschaftsberechtigten „01.03.1932” ein, berechnet das Programm (nach der uberschreibbaren Voreinstellung) das Ende der Betriebszugehörigkeit auf den „29.2.1997”. Dieses vom Programm selbst errechnete Datum wird aber beim Fortfahren in der Berechnung nicht akzeptiert. Der Benutzer wird ermahnt, ein $\mathrm{Da}$ tum im gewohnten Format „z. B. 28.10.1980!" einzugeben. Erst nach einer Korrektur des vom

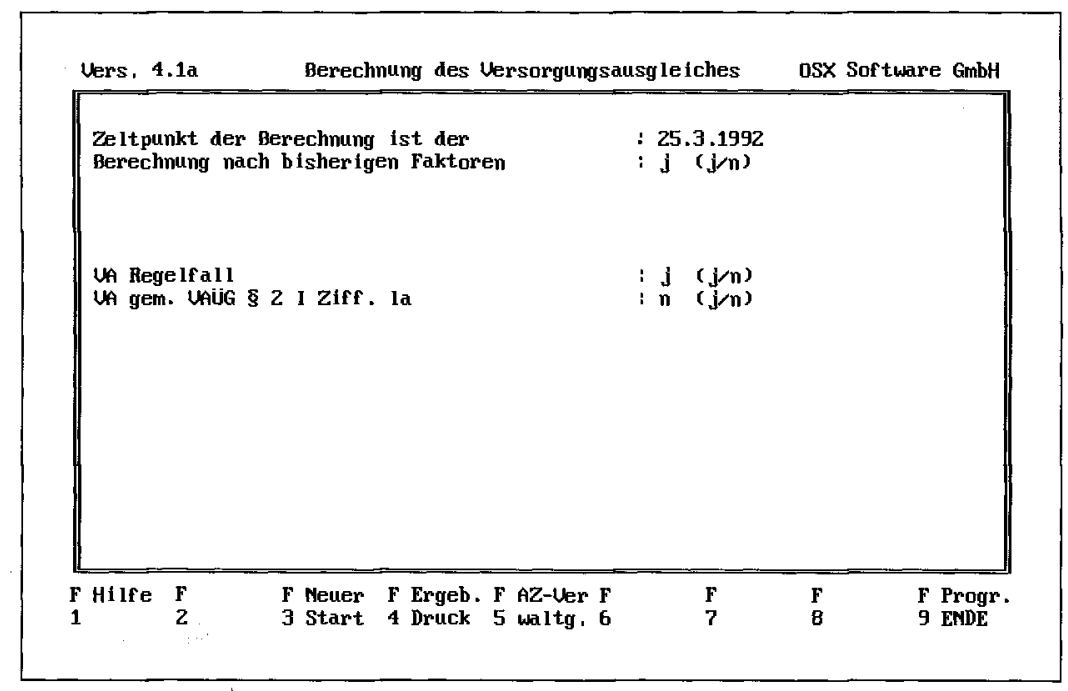

Abb. 1: Eingabebildschirm

Programm selbst fehlerhaft bestimmten Datums, etwa auf den 28.2.1997, ging es in der Berechnung weiter.

\section{Hilfemenü}

Im aufgerufenen Hilfemenü, das im übrigen ausreichend instruktiv ist und, soweit überprüft, umfassend, verständlich und auch juristisch zutreffend belehrt, befinden sich einige deftige Rechtschreibfehler. Sicherlich sind dies Marginalien, jedoch im Hinblick darauf, daß ein Rechenprogramm für den Versorgungsausgleich mit besonders sensiblem Zahlenmaterial $\mathrm{zu}$ tun hat, lassen sie leises Unbehagen aufkommen, das allerdings bei den recht umfangreichen Testrecherchen keine weitere Nahrung gefunden hat. Bei Eingabe der Beträge der Anwartschaften muß jeweils das Komma getippt werden, obwohl es sich bereits auf der Eingabemaske befindet. $\mathrm{Ab}$ Version 4.1 benutzt das Programm auch bei den von ihm errechneten Dezimalzahlen Kommatas anstelle der noch in der Version 4.0 verwendeten Punkte.

Hat man sich bei einer nichtdy namischen Anwartschaft (Abb. 2) bereits für die Dynamisierung (Abb. 3, nächste Seite) entschieden und bedient dann die Funktionstaste $\langle\mathrm{F} 8\rangle$, die mit „zurück" belegt ist, erscheint nicht etwa die vorangegangene Eingabe, sondern der - überraschende Hinweis, man habe den Dynamisierungsvorgang verlassen, und man solle die Daten neu eingeben. Das kann bei umfangreichem Zahlenmaterial, ohnehin handelt es sich beim Versorgungsausgleich fast immer um „kniffelige” Zahlen, recht ärgerlich sein.

Ist man am Ende einer Berechnung angelangt, geht es über $<F 8>$

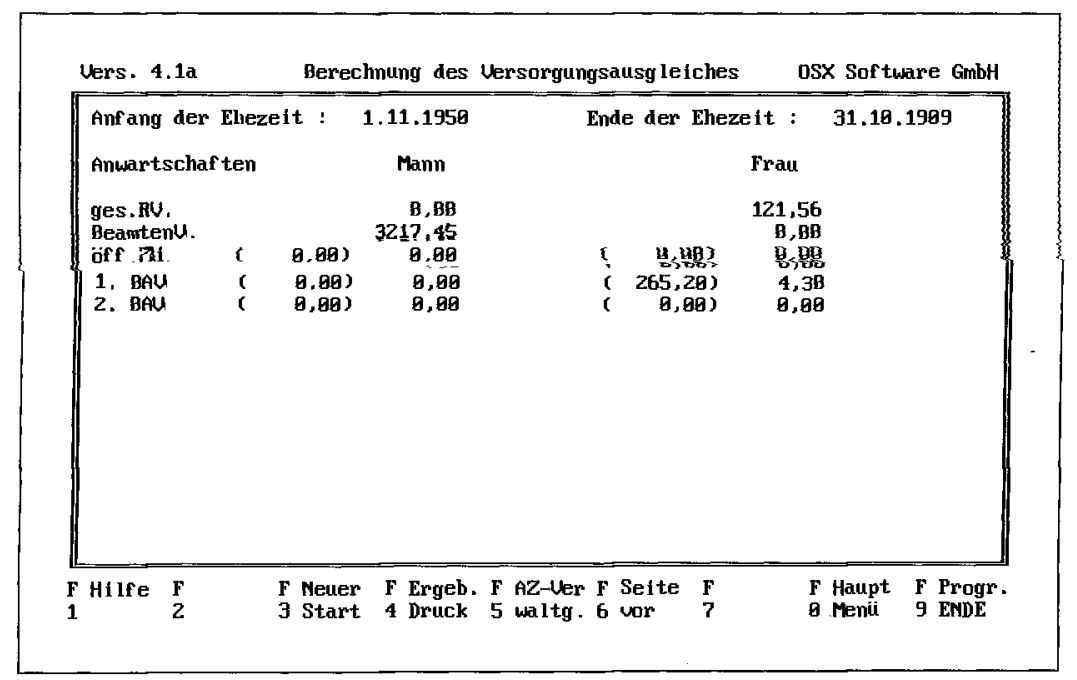

Abb. 2: Eingabe der Anwartschaften 

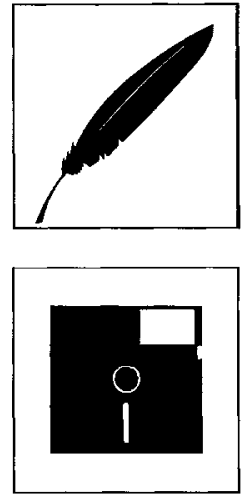

Abb 3: Dynamisierung der Anwartschaften aus der betrieblichen Altersversorgung

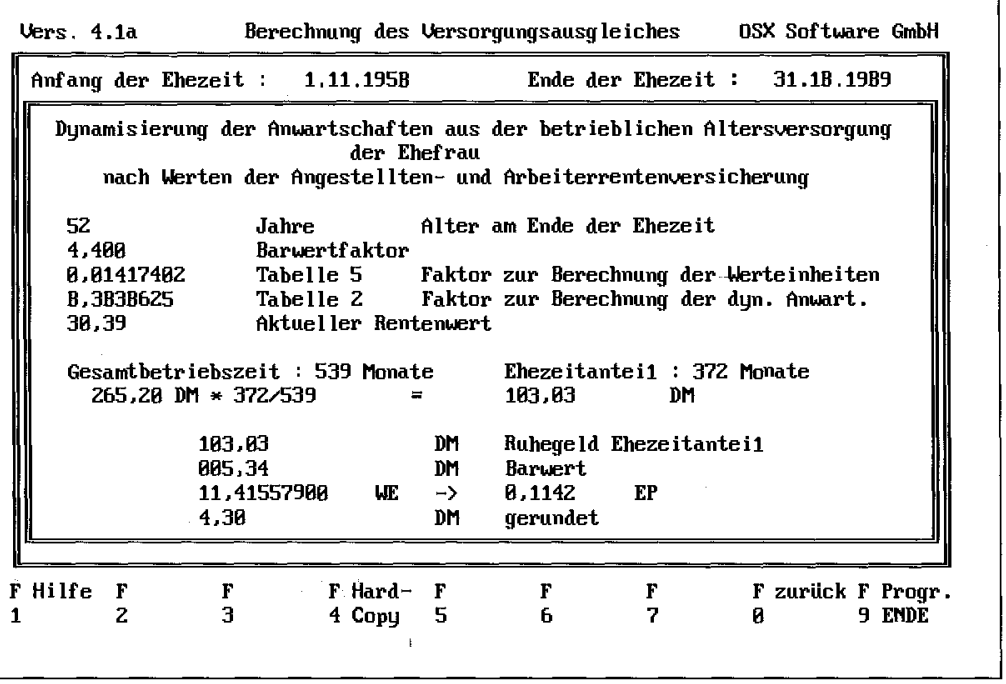

zurück zum Hauptmenü. Mit der $<$ Enter>-Taste kann man sich dann den gesamten Berechnungsvorgang nochmals Schritt für Schritt auf den Bildschirm holen und eventuell Korrekturen anbringen, was allerdings dann selbstverständlich zu einer Neuberechnung $a b$ diesem Punkt führt.

\section{Tenorierungsvorschlag}

Das Programm ist ein Rechenprogramm für Juristen. Es kann und soll dem Anwender nicht die erforderliche rechtliche Beurteilung des zur Entscheidung anstehenden Sachverhalts abnehmen. Was berechnet werden soll, richtet sich selbstredend nach dem, was der Anwender eingibt. Auf die eingegebenen Daten wendet das Programm die durch Pro- grammierung vorgegebenen $\mathrm{Ta}$ bellen der Rechengrößen an und führt die erforderlichen Rechenoperationen durch, stellt das Ergebnis dar und schlägt aufgrund dieses Ergebnisses einen Tenor vor (Abb. 4), der entsprechend $\int \$ 1587$ b IV BGB n. F., 3 I Nr. 5 VAÜG klarstellt, ob die auszugleichenden Anwartschaften in Entgeltpunkte oder in Entgeltpunkte (Ost) umzurechnen sind.

\section{Splitting}

Wird ein weiterer Ausgleich erforderlich, schlägt das Programm als weitere Optionen das analoge Quasi-Splitting, das Super-Splitting, das erweitere Quasi-Splitting sowie die Beitragsentrichtung vor und führt entsprechend der ausgeübten Option die erforderlichen Berechnungen durch.

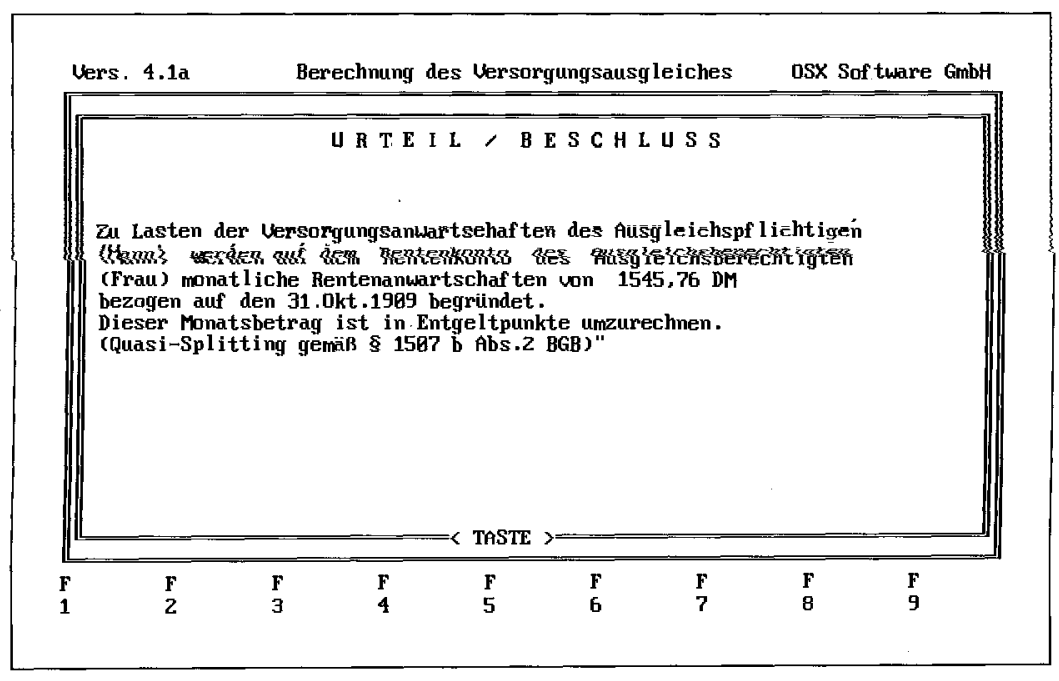

Im Falle des $₫ 1$ III VAHRG belehrt es'den Anwender im Hilfemenü, daß nach dem Gesetz nur das analoge Quasi-Splitting in Betracht komme. Schließlich wird auch noch der jeweilige Gegenstandswert angegeben. All das läßt sich speichern, ausdrukken oder zur "Weiterverarbeitung" in eine andere Datei exportieren. $\mathrm{Ob}$ das vom Programm angebotene Layout gefällt, ist Geschmacksfrage. Wie ausgeführt, läßt es sich weiterbearbeiten und dabei auch dem jeweiligen „Gerichtsritus” anpassen.

\section{Transparenz und Systemoffenheit}

Hinsichtlich der generellen Hauptanforderungen an alle $\mathrm{Re}-$ chensysteme für Juristen, nämlich Offenheit des Systems und Transparenz des Lösungsweges (vgl. zu diesen Anforderungen statt aller Viefhues, jur-pc 8/89, S. 294, 295) bleiben gegenüber dem Programm jedoch einige Vorbehalte.

In diesem Sinn ist das Programm vom System her nur "teilweise offen". Es legt den Anwender zwar nicht völlig unnachgiebig auf einen bestimmten Lösungsweg fest, liefert auch nicht nur Ergebnisse, sondern zeigt den Lösungsweg mit den erforderlichen Zwischenwerten und den sie ergebenden Faktoren, so daß das gefundene Ergebnis nachvollzogen werden kann. Selbstverständlich kann man sich etwa bei der Eingabe des Barwertfaktors über die verschiedenen Möglichkeiten informieren und die zutreffend erscheinende auswählen. Die Berechnungstabellen

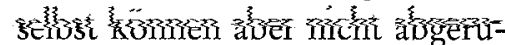
fen werden. Es gibt daher keinen Gesamtüberblick über sie und auch keine ausreichende allgemeine Kontrolle darüber, aus welcher Tabelle das Programm jeweils die Rechengrößen entnimmt, mit denen es die Berechnungen durchführt. Eine Auswahl ist - abgesehen davon, daß, wie eingangs schon erwähnt, der 
Versorgungsausgleich auch gemäß $\$ 2$ I Ziff. 1 a VÜG berechnet werden kann - nur ganz allgemein zwischen den „bisherigen”, bis zum 31.12.1991 geltenden und den „neuen" Werten möglich. Daß dies für ein juristisches Rechenprogramm nicht unbedingt ausreicht, läßt sich gerade hier besonders anschaulich aufzeigen. Allerdings ist es dabei nicht $\mathrm{zu}$ vermeiden, etwas näher auf die nicht ganz einfache Rechtsmaterie „Versorgungsausgleich", wie sie sich nach der am 01.01.1992 in Kraft getretenen Neuregelung darstellt, einzugehen.

\section{Rechtsmaterie „Versorgungsausgleich”}

Die dem Rezensenten zunächst vorliegende Programmversion 4.0 legte bei der Dynamisierung von nichtdynamischen Versorgungsanwartschaften, wenn das Ende der Ehezeit mit einem Datum vor dem 01.01.1992 eingegeben worden war, die alten Rechengrößen zugrunde. Die auf Anfrage vom Verlag übersandte ver. besserte Version 4.1 zieht, so die Verlagsinformation, „standardmäßig” die sogenannten BfAFaktoren - die alten Rechengrößen bleiben als Option erhalten - heran und schließt sich damit der nunmehr wohl herrschenden, für das alte Recht auch vom BGH in ständiger Rechtsprechung vertretenen Meinung (vgl. BGH, NJW 1986, 1189; 1989, 29, 32, 34; FamRZ 1989, $153 ; 492 ; 1060)$ an, daß es für die Frage, welche gesetzlichen Bestimmungen maßgebend sind, auf den Zeitpunkt der Entscheidung ankommt und geht also bei seinen Berechnungen von den neuen Rechengrößen aus. Da hier wohl im Gesetz eine Regelungslücke vorliegt (so Bergner, NJW 1992, 479, 490, Glockner, FamRZ 1992, 149, 150), ist diese "herrschende" Meinung, obwohl sie vieles für sich hat, keineswegs zwingend. Eine Gegenmeinung könnte etwa darauf verweisen, daß der Ge- setzgeber trotz der Neuformulierung des $₫ 1587$ a II Nr. 2 BGB hier zur Frage der Fortgeltung der BGH-Rechtsprechung nicht Stellung genommen, während er in einem anderen Fall - Ermittlung des auszugleichende Anrechts bei bereits gezahlten Renten - in der Begründung ausdrücklich darauf hingewiesen hat, daß „die von der Rechtsprechung des BGH aufgestellte Regel nicht berührt" wird (vgl. Ruland, NJW 1992, $77 \mathrm{ff}, 80 \mathrm{f}$ ).

Offenbleibt nach wie vor die Frage, wie hinsichtlich der vorläufigen Dürchschnittsentgelte für die Zeit vor $1991 \mathrm{zu}$ verfahren ist, die der Gesetzgeber bislang nicht festgesetzt hat. Hier folgt das Programm, soweit feststellbar, ohne eine Option zu lassen, in der Version 4.1 der von Bergner, a. a. O., S. 490, aufgezeigten Lösungsmöglichkeit, die auch von Glockner, a. a. O., und Kemnade, FamRZ 1992, 151 f, vertreten wird. Gerade Bergner, a. a. O., zeigt aber besonders anschaulich, daß auch ganz andere Wege denkbar sind.

\section{Geringer \\ Entscheidungsfreiraum}

Eine umfassende Transparenz durch Abruf des gesamten angewendeten Tabellenwerks wäre auch deshalb besonders wichtig, weil zur Zeit - März 1992 - noch weitgehend Unsicherheit darüber besteht, welche Rechengrößen jeweils „richtig” sind (vgl. hierzu Ruland, NJW 1992, 77 ff, 78; Bergner, a. a. O., 490; Glockner, a. a. O., 150; Kemnade, a. a. O.). Die Situation wird noch weiter dadurch kompliziert, daß die von einzelnen Autoren yerïffastieh ten "nichtamtlichen” Rechengrößen auch rein numerisch offenbar nicht unbedingt unumstößlich sind. So hat Bergner seine a. a. O. veröffentlichten Tabellen der Umrechnungsfaktoren auf der Grundlage des endgültigen Durchschnittsentgelts nachträglich in vier Werten geändert (vgl. NJW 1992, 482 f, 674). So- weit durch umständliche Einzelabfrage über fingierte Fälle festgestellt werden konnte, geht das Programm ebenfalls von diesen geänderten Werten aus. Wegen des Fehlens einer aus dem Programm abrufbaren umfassenden Information über die Tabellen sowie einer weitergehenden als der vom Programm eingeräumten Option zwischen bisherigen und neuen Werten für bestimmte der aufgezeigten Alternativen durch Vorabauswahl der Tabellen, hat der Anwender nur bei einiger Anstrengung den erforderlichen „Freiraum” für seine Entscheidung. Schließlich fehlt, weil die Tabellen nicht aufgerufen werden können, auch die Möglichkeit, eventuelle numerische Fehler im Tabellenwerk des Programms, die sich auch bei noch so großer Sorgfalt des Software-Herstellers erfahrungsgemäß immer wieder einschleichen können, etwa durch Abgleichen mit einer externen „amtlichen" Tabelle, bereits von vornherein zu erkennen beziehungsweise deren Vorhandensein auszuschließen. Einem (sehr?) gewissenhaften Anwender bleibt nur die Überprüfung in jedem Einzelfall, wie es der Rezensent getan hat, wodurch die Zeitersparnis durch die Benutzung des Programms nicht unerheblich verringert werden dürfte. Eine Gesamtabgleichung, etwa im Zusammenhang mit der Programminstallierung, wäre zwar auch recht zeitraubend, würde aber dafür nur einmal anfallen.

\section{Keine Aufrufbarkeit des Tabellenwerks}

Aufrufbarkeit des vom Pro-

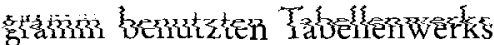
und Auswahlmöglichkeit unter den verschiedenen Tabellen wären sicherlich kein unüberwindliches technisches Problem, wie insbesondere zahlreiche Programme zur Unterthaltsberechnung zeigen, bei denen der Anwender in der Regel sowohl die einzelnen Tabellen aufrufen, als auch unter diesen auswählen kann.
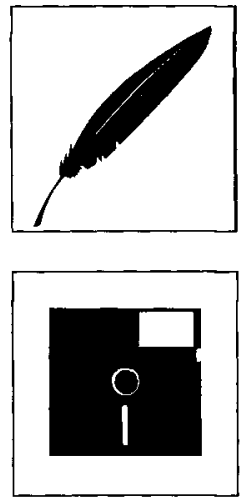

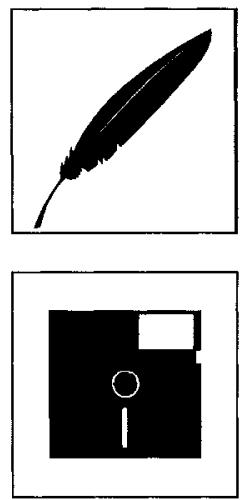

der konservativen Taschenrechnermethode erhebliche Zeit sparen. Das lästige und zeitraubende Eingeben der Rechengrößen mit den damit verbundenen Fehlerquellen entfällt. Die Ergebnisse erscheinen klar und übersichtlich dargestellt auf dem Bildschirm. Das alles mag verlockend klingen. Aber das Programm und seine Anwendung allein machen aus dem Anwender noch keinen Experten im Versorgungsausgleich. Wer die Rechtsmaterie Versorgungsausgleich nicht beherrscht, wird mit keinem Programm, natürlich auch nicht mit diesem, eine Ausgleichsberechnung mit Erfolg durchführen können. Vor solchen unrealistischen Vorstellungen sei gewarnt. Wer jedoch vom Versorgunsausgleich etwas versteht, wird das Programm schnell anzuwenden lernen und von der damit verbundenen, nicht unbeträchtlichen Zeitersparnis profitieren. $\mathrm{Ob}$ sich dafür die Anschaffung lohnt, schließlich muß man mit mindestens einem, eventuell sogar zwei Updates pro Jahr rechnen, ist eine Frage, die jeder potentielle Erwerber für sich selbst beantworten muß. Hat man sich für die Anschaffung eines entsprechenden Programms entschieden, kommt das vorliegende, auch unter Würdigung der geäußertern Kritik, durchaus als empfehlenswert in Betracht.

und sicher. Auch ein weniger geübter Benutzer wird schon nach kurzer Eingewöhnung gegenüber

\section{II. „Unterhalt”}

Programmversion 4.1, 1. Halbjabr 1992

Nach der Programmbeschreibung im Handbuch, für dessen Aufmachung und Gestaltung die vorstehende positive Beurteilung gleichermaßen gilt, unterstützt dieses den Anwender bei der Berechnung "des bereinigten Nettoeinkommens (Abb. 5 und 6, auf der nächsten Seite) und/oder der Unterhaltsansprüche von Kindern und/oder Ehegatten". Dieser Ankündigung wird das Programm gerecht. Für das Beitrittsgebiet ist jedoch für die Berechnung des Kindesunterhalts nur die „Berliner Tabelle” (vgl. FamRZ 1991,
408) vorgesehen, nicht aber etwa die „Sächsische Unterhaltstabelle", Stand 01.02.1992 - früher "Chemnitzer Tabelle" - (vgl. DtZ 1992, 21; 117). Alle damit verbundenen Rechenoperationen, etwa auch in Mangelfällen und unter Berücksichtigung abweichender Berechnungsgrößen im Beitrittsgebiet, mit der zuvor erwähnten Einschränkung, sind mit voreingestellten Paramatern - „Düsseldorfer" und „Berliner Tabelle” können hier über Funktionstaste abgerufen und insgesamt eingesehen werden - oder mit selbst eingegebenen schnell und

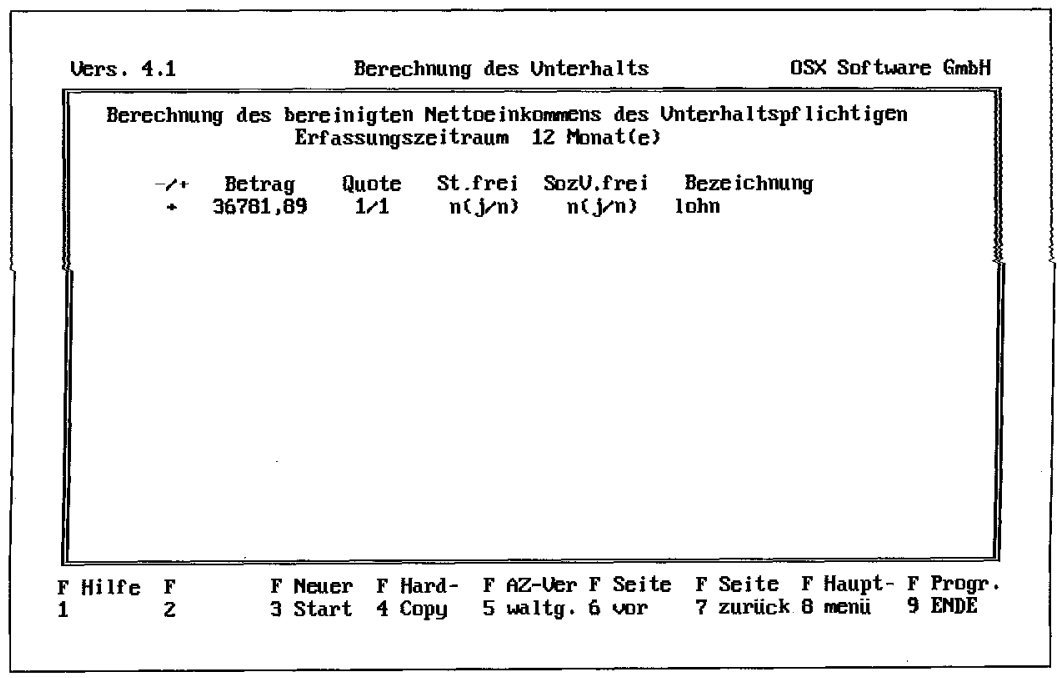

problemlos durchzuführen. Die zur Rezension stehende Programmversion 4.1 berücksichtigte bereits die rückwirkend zum 01.01.1992 in Kraft getretenen Änderun-gen - erhöhtes Erstkindergeld und erhöhter Kinderfreibetrag - und selbstverständlich für Berechnungszeiträume ab dem 01.07.1991 den Solidaritätszuschlag.

\section{Gelungene Steuerberechnung}

Gerade die Steuerberechnung verdient als besonders gelungen der Hervorhebung. Vermißt wurden Optionen zur Berechnung des Vorsorgeunterhalts und des steuerlichen Real-Splittings beim Fheyattenunerhak Da bieten einige andere Programme mehr. Die Systemoffenheit dieses Programms erscheint ausreichend, obwohl man sich auch hier noch Verbesserungen vorstellen könnnte. Es wäre wünschenswert, daß jeder einzelne Rechenschritt auf dem Bildschirm aufgezeigt und so exakt nachvollzogen werdèn könnte. 
Installation und Bedienung des Programms bieten keine besonderen Schwierigkeiten. Die beigegebenen „Readme"-Dateien informieren umfassend und verständlich über die zwischenzeitlich gegenüber dem noch nicht aktualisierten Handbuch vorgenommenen Änderungen. Weitere, eventuell noch vorhandene Unklarheiten beseitigt erfolgreich und umfassend das sehr gut gestaltete und informative Hilfemenü, das auch hier erforderlichenfalls rechtlichen Nachhilfeunterricht erteilt.

\section{Ermittlung des angemessenen Unterhalts}

Die eingegebenen (Zeit-)Daten werden auf ihre Plausibilität hin überprüft. Besonderheiten sind hierbei nicht aufgefallen. Die Optionsmöglichkeiten hinsichtlich der Berechnungsparameter (mal abgesehen von den Unterhaltstabellen für das Beitrittsgebiet, wobei nicht übersehen werden darf, daß diese sich weitgehend an der „Düsseldorfer Tabelle" orientieren) lassen keine Wünsche offen. Die Berechnungsergebnisse können ausgedruckt und/oder in eine andere Datei exportiert und dort „weiterverarbeitet" werden. All dies enspricht dem heute üblichen Standard. Vielmehr als bei Programmen zur Berechnung des Versorgungsausgleich stellt sich bei Unterhaltsberechnungsprogrammen die Frage nach dem Nutzen, besonders unter dem
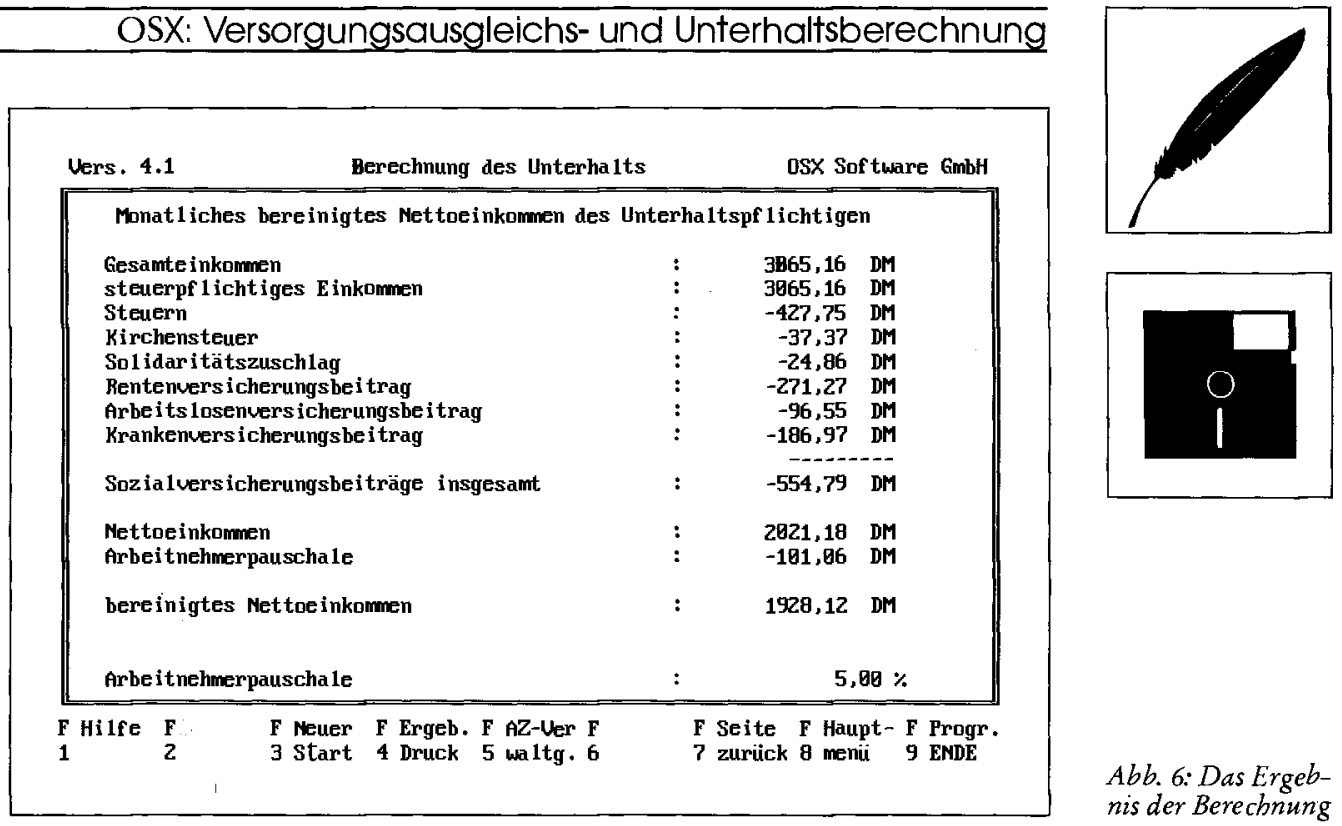

Gesichtspunkt einer KostenNutzen-Analyse etwa für einen Richter, der die Anschaffung aus seiner Privatschatulle bestreiten muß. Dabei sind auch die Updates zu berücksichtigen, die in unserer schnellebigen Zeit bei der vorliegenden Materie immer häufiger notwendig werden. So steht etwa noch im Sommer 1992 eine neue "Düsseldorfer Tabelle” ins Haus. Unterhaltsberechnungen sind in der Regel, anders als vie-le Versorgungsausgleichsberech-nungen, verhältnismäßig einfach, sieht man einmal von verzwickten Mangelfällen $a b$. Das „Zahlenmaterial” ist meist übersichtlicher, man arbeitet mit vertrauten Zahlen mit höchstens zwei Kommastellen, sofern man nicht auch auf die verzichtet. Schließlich soll man ja nach dem Gesetz den „angemessenen” Unterhalt ermitteln, dessen Kriterien zwar vielfältig, aber auch in engen Fällen kaum in den Stellen hinterm Komma zu finden sind.
Oft ist man da, Mangelfälle einmal ausgenommen, bei hinreichender Übung mit dem $\mathrm{Ta}$ schenrechner auch nicht viel langsamer als mit einem noch so komfortablen Berechnungsprogramm. Vorsorgeunterhalt und begrenztes steuerliches RealSplitting mögen davon Ausnahmen machen, aber die scheiden beim vorliegenden Programm ohnehin aus. Die wesentliche Unterstützung bietet das Programm auch dem im Unterhaltsrecht versierten Benutzer bei der Umrechnung von Brutto- in Nettobeträge. $\mathrm{Da}$ schlägt das Programm sicherlich jeden noch so geübten „Taschenrechneranwender".

Auch bei dem Programm „Unterhalt” muß letzdich jeder "Unterhaltsbeflissene" für sich selbst herausfinden, ob er mit dem Erwerb - und der Anwendung des Programms sich eine mehr oder minder große Zeitersparnis erkaufen möchte. 